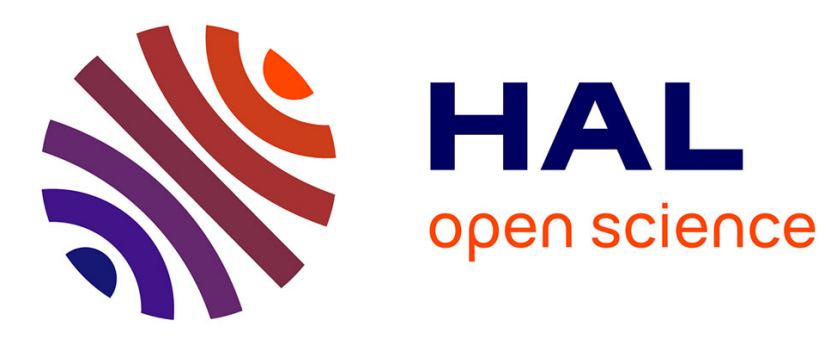

\title{
Relaxed stability conditions for Takagi-Sugeno fuzzy systems
}

Mohammed Chadli, Didier Maquin, José Ragot

\section{To cite this version:}

Mohammed Chadli, Didier Maquin, José Ragot. Relaxed stability conditions for Takagi-Sugeno fuzzy systems. IEEE International Conference on Systems, Man, and Cybernetics, SMC'2000, Oct 2000, Nashville, United States. pp.3514-3519, 10.1109/ICSMC.2000.886553 . hal-00201428

\section{HAL Id: hal-00201428 \\ https://hal.science/hal-00201428}

Submitted on 17 Apr 2014

HAL is a multi-disciplinary open access archive for the deposit and dissemination of scientific research documents, whether they are published or not. The documents may come from teaching and research institutions in France or abroad, or from public or private research centers.
L'archive ouverte pluridisciplinaire HAL, est destinée au dépôt et à la diffusion de documents scientifiques de niveau recherche, publiés ou non, émanant des établissements d'enseignement et de recherche français ou étrangers, des laboratoires publics ou privés. 


\title{
Relaxed stability conditions for Takagi-Sugeno fuzzy systems
}

\author{
Mohammed CHADLI, Didier MAQUIN, José RAGOT \\ Centre de Recherche en Automatique de Nancy, CNRS , UPRESA 7039 \\ 2, Avenue de la forêt de Haye, 54516 Vandoeuvre les Nancy - France. \\ Phone: (33) 3835959 59, Fax: (33) 383595644 \\ Email : \{Mohamed.Chadli, Didier.Maquin, José.Ragot\}@ensem.inpl-nancy.fr
}

\begin{abstract}
This paper discusses conditions on stability and stabilization of continuous T-S fuzzy systems. Stability analysis is derived via non-quadratic Lyapunov function technique and LMIs (Linear Matrix Inequalities) formulation to obtain an efficient solution. The nonquadratic Lyapunov function is built by inference of quadratic Lyapunov function of each local model. We show that stability condition of the open-loop T-S systems is assured under certain restrictions on the rate of change of state variables. Following a similar approach, stabilization of the closed-loop continuous T-S fuzzy systems using the well-known PDC (Parallel Distributed Compensation) technique is investigated. The design methodology is illustrated by numerical examples.
\end{abstract}

\section{Introduction}

Since last years many works have been focused on the stability and the stabilisation of closed loop fuzzy systems including a fuzzy controller. Specially, the approach using T-S fuzzy models [5], considered like a universal approximator [8], has been considered extensively. The TS fuzzy models are described by a set of fuzzy "If ... then" rules with fuzzy sets in the antecedents and dynamics LTI systems in the consequent. These sub-models are considered as local linear models, the aggregation of which representing the nonlinear system behaviour.

The issue of stability and stabilisation of T-S fuzzy control systems has been considered extensively in nonlinear frameworks. However, the present results are only sufficient and require conservative assumptions. For example, Tanaka and Sugeno presented sufficient conditions for the stability of T-S models [6] using a quadratic Lyapunov approach. The stability depends on the existence of a common positive definite matrix guarantying the stability of all local subsystems. These stability conditions may be expressed in linear matrix inequalities (LMIs) form [10]. The obtaining of a solution is then facilitate by using numerical toolboxes for solving such problems. Recently LMI constraint has been added to compute a decay rate and to guarantee that the control law action doesn't permit an exceeding of a pre-defined normbound of input and output signals [2][9]. However, if the number of sub-models is large, it might be difficult to find a common matrix. Moreover, these constraints are often very conservative and it's well known that, in a lot of cases, a common positive definite matrix does not exist, whereas the system is stable.

To overcome this limitation, some works have been developed in order to establish new stability conditions by relaxing some of the previous constraints. So one way for obtaining relaxed stability conditions consists to use a piecewise quadratic Lyapunov function formulated as a set of LMIs [3]. Using the PI fuzzy controller and the Lyapunov technique, the authors in [13] show that asymptotic stability of the Takagi-Sugeno fuzzy systems can be ensured under certain restriction on the control signal and the rate of change of the output. Jadbabaie in [1] introduces T-S fuzzy rules that describe the Lyapunov function where each T-S rule has fuzzy sets in the antecedents and quadratic Lyapunov function in the consequent. The global Lyapunov function is state dependant. This method requires fixing a priori bound on the variation of the state, to prove the stability of the T-S fuzzy systems [1]. The major disadvantage of this approach is the fact that a systematic way to pick the bound of state variable vector has not been derived. The author only proposes to pick initial large number for this bound and then iteratively reducing this value by a trial and error method.

The goal of this paper is double. Using the non-quadratic Lyapunov function, we will derive sufficient condition for stability and also stabilisation of the continuous T-S fuzzy system.

This paper is organised as follows: section 2 presents an overview of T-S fuzzy systems. In section 3 we recall the LMI formulation of basic quadratic stability conditions. In section 4, first a fuzzy Lyapunov function is built by inference of the quadratic Lyapunov functions designed for each local model. We also develop a new proposition to take into account a finite number of rules activated in each time for less of conservatism. At last, using the LMI technique for efficient way of resolution, we develop a method to estimate an upper bound of the variation of the state to prove the stability of T-S continuous fuzzy systems. In section 5, using the concept of PDC to perform control laws and LMIs formulation, similar approach is used for the stabilization of T-S fuzzy closed loop system. In both cases numerical examples are given to illustrate the effectiveness of the proposed stability conditions. 


\section{Takagi-Sugeno fuzzy systems}

The T-S fuzzy models are described by a set of fuzzy "if...then" rules with fuzzy sets in the antecedents and dynamic LTI systems in the consequent. These sub-systems represent local linear input-output relations of a nonlinear systems. A general T-S plant rule can be written as follows:

$i^{\text {th }}$ rule :

IF $x_{1}(t)$ is $\mathrm{F}_{1}^{\mathrm{i}}$ and..., $x_{n}(t)$ is $\mathrm{F}_{\mathrm{n}}^{\mathrm{i}}$ THEN $\dot{x}(t)=A_{i} x(t)+B_{i} u(t)$

Where $n$ is the number of rules (sub-models), $x(t) \in \mathfrak{R}^{p}$ is the state vector, $y(t) \in \mathfrak{R}^{l}$ is the output vector, $u(t) \in \mathfrak{R}^{m}$ is the input vector, $A_{i} \in \Re^{p x p}, B_{i} \in \Re^{p x m}$.

The global T-S fuzzy systems is inferred as follows:

$$
\dot{x}(t)=\frac{\sum_{i=1}^{n} \grave{u}_{i}(x(t))\left(A_{i} x(t)+B_{i} u(t)\right)}{\sum_{i=1}^{n} \grave{u}_{i}(x(t))}
$$

where $\grave{u}_{i}(x(t))=\prod_{j=1}^{n} f_{j}^{i}\left(x_{j}(t)\right)$

and $f_{j}^{i}\left(x_{j}(t)\right)$ is the membership grade of $x_{j}(t)$ to the fuzzy set $\mathrm{F}_{\mathrm{j}}^{\mathrm{i}}$.

Finally we define:

$$
\mu_{i}(x(t))=\frac{\grave{u}_{i}(x(t))}{\sum_{i=1}^{n} \grave{u}_{i}(x(t))}
$$

$\mu_{i}(x(t))$ is the normalised membership function in relation with the $i^{\text {th }}$ sub-system such that:

$$
\left\{\begin{array}{l}
\sum_{i=1}^{n} \mu_{i}(x(t))=1 \\
0 \leq \mu_{i}(x(t)) \leq 1 \quad \forall i: 1 . . n
\end{array}\right.
$$

Equation (1) can be rewriten as:

$$
\dot{x}(t)=\sum_{i=1}^{n} \mu_{i}(x(t))\left(A_{i} x(t)+B_{i} u(t)\right)
$$

It is assumed in this paper that the membership functions depend only on the state of the system and that only $r$ locals models are activated for a given $x(\mathrm{t})$ (the another $(n-r)$ local models are ignored).
The minimum and maximum eigenvalues of the matrix $X$ will be respectively denoted by $\lambda_{m}(X)$ and $\lambda_{M}(X)$.

\section{Basic stability conditions}

The open-loop T-S system of (4) is defined as:

$$
\dot{x}(t)=\sum_{i=1}^{n} \mu_{i}(x(t)) A_{i} x(t)
$$

Let us recall first the basic stability conditions of the openloop system (5) derived using quadratic Lyapunov function.

Theorem [6]: The continuous fuzzy system described by (5) is globally asymptotically stable if there exists a common $P=P^{T}>0$ such that:

$$
A_{i}^{T} P+P A_{i}<0 \quad \forall i: 1 . . n
$$

The existence of such a common positive definite matrix described by LMIs (6) is a key to check the global stability of T-S continuous fuzzy system.

The authors in [12] propose an analytic way to finding a common $P$ in the particular case where the matrices $A_{i}$ are asymptotically stable and commute pairwise.

Theorem[12]: For a given positive definite matrix $Q$, let $P_{1}, \ldots, P_{n}$ be the unique solution of the following Lyapunov equations:

$$
\begin{aligned}
& A_{1}^{T} P_{1}+P_{1} A_{1}=-Q \\
& A_{i}^{T} P_{i}+P_{i} A_{i}=P_{i-1} \quad \forall i: 2 . . n
\end{aligned}
$$

If the following assumption is made:

$$
A_{i} A_{j}=A_{j} A_{i} \quad \forall i, j: 1 . . n
$$

i. e. all matrices commute pairwise, then the T-S fuzzy system (5) is globally asymptotically stable and have as a common lyapunov function $V(x)=x^{T} P_{n} x$

Proof: see [12]

These results mean that a sufficient condition for the existence of a common $P$ is that each $A_{i}$ must be asymptotically stable and commute pairwise.

It is also shown that a common $P$ exists if the following conditions hold [11]:

$$
A_{i}+A_{j}<0 \quad \forall i, j: 1 . . n
$$

The proof can be easily obtained by summing the LMIs (6). 
This result means that a necessary condition for the existence of a common $P$ is that each $A_{i}$ and $\left(A_{i}+A_{j}\right)$ must be stable.

These above conditions show clearly the conservativness of the method, i.e. we can find T-S fuzzy systems that are stable but there is no common $P$ to prove the stability.

\section{Stability analysis using non-quadratic Lyapunov function}

So one way for obtaining relaxed stability conditions and overcome these limitations consists to use a non-quadratic Lyapunov function candidate defined as:

$\mathrm{i}^{\text {th }}$ rule :

IF $x_{1}(t)$ is $\mathrm{F}_{1}^{\mathrm{i}}$ and..., $x_{n}(t)$ is $\mathrm{F}_{\mathrm{n}}^{\mathrm{i}}$ THEN $V_{i}(x(t))=x^{T} P_{i} x$

where each T-S rule has the same fuzzy sets as the fuzzy system in the antecedents and quadratic Lyapunov functions in the consequent.

Using the same inference as before we obtain the following state dependent Lyapunov function:

$$
V(x)=x^{T} P(x) x
$$

with $P(x)=\sum_{i=1}^{n} \mu_{i}(x(t)) P_{i} \quad$ and $\quad P_{i}=P_{i}^{T}>0$

where $\mu_{i}(x(t))$ has the properties (3).

The derivative of this Lyapunov function is:

$$
\dot{V}(x(t))=\dot{x}(t)^{T} P(x) x(t)+x(t)^{T} P(x) \dot{x}(t)+x(t)^{T} \dot{P}(x) x(t)
$$

Proposition 1: given a matrix as in (11), one can bound the last term of (12) as follows:

$$
x(t)^{T} \dot{P}(x) x(t) \leq \gamma\|\dot{x}(t)\|\|x(t)\|^{2} \sum_{i=1}^{r} \lambda_{M}\left(P_{i}\right)
$$

with:

$$
\gamma=\operatorname{Max}_{i=1}^{n}\left(\left\|\frac{\partial \mu_{i}(x(t))}{\partial x(t)}\right\|\right)
$$

$r$ is the number of rules activated simultaneously at each time such that $2 \leq r \leq n$ and the eigenvalues are ordered such that:

$$
\lambda_{M}\left(P_{1}\right)>\ldots>\lambda_{M}\left(P_{r}\right)>\ldots>\lambda_{M}\left(P_{n}\right)
$$

Proof: The proof follows from bounding the derivative of the normalized membership functions and considering the properties of the largest eigenvalue of a symmetric matrix [14]
Theorem 1: Suppose that there exist $Q$ and $P_{i}$ such that the following LMIs hold:

$$
\begin{aligned}
& \forall i: 1 . . n \quad A_{i}^{T} P_{i}+P_{i} A_{i}<-Q \text { and } \\
& \forall i \neq j: 1 . . n \quad \frac{A_{i}^{T} P_{j}+P_{j} A_{i}+A_{j}^{T} P_{i}+P_{i} A_{j}}{2}<-Q \\
& \text { such that } \mu_{i}(x(t)) \mu_{j}(x(t)) \neq 0
\end{aligned}
$$

Then the global model (5) is exponentially stable if the following constraint on the variation of the state is satisfied:

$$
\|\dot{x}(t)\| \leq \frac{\lambda_{M}(Q)}{\gamma \sum_{i=1}^{r} \lambda_{M}\left(P_{i}\right)}
$$

Proof: The proof is obtained by computing the derivative of the Lyapunov function (12) along the trajectory of the T-S fuzzy system (5) and takes into account the proposition 1. The exponential stability is proved by considering the properties of the largest and the smallest eigenvalues of symmetric matrix [14].

\section{Example}

In order to appreciate the efficiency of the proposed method, we consider example given in [1] where the T-S fuzzy model is composed of two rules.

rule 1: IF $x_{1}(t)$ is $\mathrm{F}_{1}^{1}$ THEN $\dot{x}(t)=A_{1} x(t)$

rule 2 : IF $x_{1}(t)$ is $\mathrm{F}_{1}^{2}$ THEN $\dot{x}(t)=A_{2} x(t)$

The fuzzy sets are described by the following two triangular membership functions:

$\mu_{1}(x(t))=1-\frac{\left|x_{1}(t)\right|}{3}, \quad \mu_{2}(x(t))=1-\mu_{1}(x(t))$

and:

$$
A_{1}=\left[\begin{array}{ll}
-5 & -4 \\
-1 & -2
\end{array}\right], A_{2}=\left[\begin{array}{cc}
-2 & -4 \\
20 & -2
\end{array}\right]
$$

the global T-S fuzzy model is:

$$
\dot{x}(t)=\left(\mu_{1}(x(t)) A_{1}+\mu_{2}(x(t)) A_{2}\right) x(t)
$$

Note that a common $P$ satisfying (6) that proves the stability of the system (15) does not exist whereas the simulation results indicate that this system is asymptotically stable.

Stability conditions in theorem 1 give the following LMIs: 


$$
\begin{aligned}
& A_{1}^{T} P_{1}+P_{1} A_{1}<-Q \\
& A_{2}^{T} P_{2}+P_{2} A_{2}<-Q \\
& \frac{A_{1}^{T} P_{2}+P_{2} A_{1}+A_{2}^{T} P_{1}+P_{1} A_{2}}{2}<-Q
\end{aligned}
$$

By solving LMIs (16) the following values are obtained:

$$
P_{1}=\left[\begin{array}{ll}
0.77 & 0.20 \\
0.20 & 0.51
\end{array}\right], P_{2}=\left[\begin{array}{ll}
2.86 & 0.10 \\
0.10 & 0.44
\end{array}\right], Q=\left[\begin{array}{ll}
5.49 & 3.08 \\
3.08 & 1.74
\end{array}\right]
$$

Then the inequality (14) allows to deduce the conditions of stability, namely the upper bound of the state variation of the system:

$$
\|\dot{x}(t)\| \leq 7.64
$$

The figure 1 shows that, with respect of the constraint (17), all trajectories of the above fuzzy systems converge to zero.
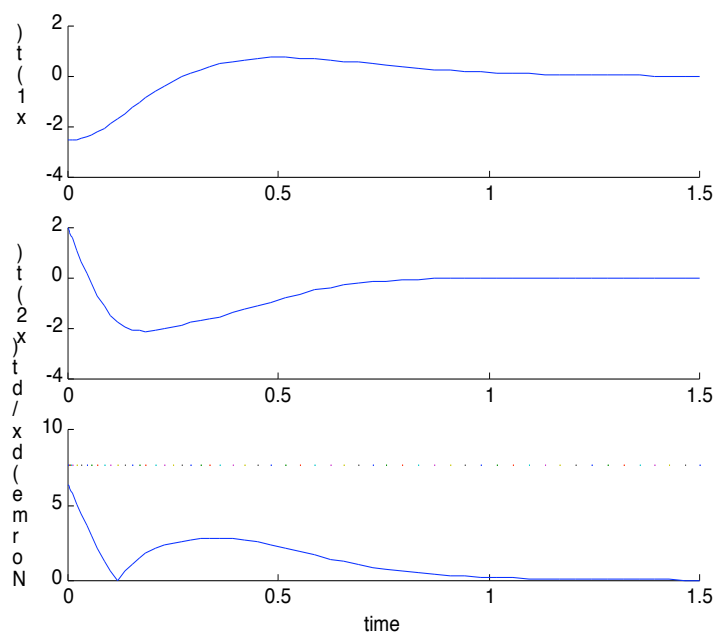

Figure 1: States trajectories of (15) with initials values $x(0)=\left[\begin{array}{ll}-2.5 & 2\end{array}\right]$

\section{Non-quadratic stabilizability}

In this section we utilise the PDC technique [7][2][4] to design fuzzy controllers to stabilize fuzzy system (4). In the PDC approach, the consequent part is local linear feedback law and the antecedent part shares the same fuzzy sets as the fuzzy system.

$\mathrm{i}^{\text {th }}$ rule :

IF $x_{1}(t)$ is $\mathrm{F}_{1}^{\mathrm{i}}$ and..., $x_{n}(t)$ is $\mathrm{F}_{\mathrm{n}}^{\mathrm{i}}$ THEN $u(t)=-K_{i} x(t)$

the resulting global fuzzy controller, which is nonlinear in general, is:

$$
u(t)=-\sum_{i=1}^{n} \mu_{i}(x(t)) K_{i} x(t)
$$

where $\mu_{i}(x(t))$ is defined in (3).

Substituting (18) in (4), we obtain the closed-loop T-S continuous fuzzy system:

$$
\dot{x}(t)=\sum_{j=1}^{n} \sum_{i=1}^{n} \mu_{i}(x(t)) \tilde{A}_{i j} x(t)
$$

where $\widetilde{A}_{i j}=A_{i}-B_{i} K_{j}$

\subsection{Stabilizability analysis using a non-quadratic Lyapunov function}

To study the stability of the closed-loop T-S fuzzy system (19) and find appropriate $K_{i}$ gains, we need the following propositions to prove theorem 2 .

Proposition 2 : given normalized membership functions as described in (3), the following inequality holds:

$$
\sum_{i \neq j}^{n} \mu_{i}(x(t)) \mu_{j}(x(t)) \leq 1-\frac{1}{r} \quad \forall 2 \leq r \leq n
$$

where $(r)$ is the number of rules activated simultaneously at each time.

Proof: the proof follows from the properties of the normalized membership function (3) and the corollary 4 in [2].

Proposition 3: given normalized membership functions as described in (3), the following inequality holds:

$$
\begin{aligned}
& \sum_{i=1}^{n} \mu_{i}(x(t))^{3} \geq \frac{1}{r}-\sum_{i \neq J}^{n} \mu_{i}(x(t)) \mu_{j}(x(t))^{2} \\
& \forall 2 \leq r \leq n
\end{aligned}
$$

Proof: the proof follows from the proposition 2 and the properties of the normalized membership function (3).

In the following, for less of conservatism, it is assumed that only two rules (sub-models) are activated simultaneously at each time, assumption easy to realise in the case of triangular and trapezoidal normalized memberships functions.

Let us now define the following matrices:

$$
H_{i j k}=\widetilde{A}_{i j}^{T} P_{k}+P_{k} \widetilde{A}_{i j}
$$

Theorem 2: Suppose that there exist $Q$ and $P$ such that the following LMIs hold: 


$$
\begin{gathered}
\forall i: 1 . . n \quad H_{i i i}<-Q \text { and } \\
\forall i, j: 1 . . n \quad H_{i j i}+H_{j i i}+H_{i j}<-Q
\end{gathered}
$$

such that: $\mu_{i}(x(t)) \mu_{j}(x(t)) \neq 0$

Then the global model (19) is exponentially stable if the following constraint on the variation of the state is satisfied:

$$
\|\dot{x}(t)\| \leq \frac{\lambda_{M}(Q)}{\gamma r \sum_{i=1}^{r} \lambda_{M}\left(P_{i}\right)}
$$

Proof: The proof is obtained by computing the derivative of the Lyapunov function (12) along the trajectory of the T-S fuzzy system (19) and takes into account the proposition 1 and proposition 3 .

The LMIs design (23) allows to obtain simultaneously the controller feedback gains $K_{i}, Q$ and $P_{i}$ matrices and then compute the constraint (24) which ensure the exponential stability.

\subsection{Numerical example}

Consider the following T-S fuzzy model composed of two rules:

rule 1: IF $x_{1}(t)$ is $\mathrm{F}_{1}^{1}$ THEN $\dot{x}(t)=A_{1} x(t)+B_{1} u(t)$

rule 2: IF $x_{1}(t)$ is $\mathrm{F}_{1}^{2}$ THEN $\dot{x}(t)=A_{2} x(t)+B_{2} u(t)$

where $\mathrm{F}_{1}^{1}$ and $\mathrm{F}_{1}^{2}$ are fuzzy sets described by the following two triangular membership functions:

$$
\mu_{1}(x(t))=\frac{1+x_{1}(t)}{2}, \quad \mu_{2}(x(t))=1-\mu_{1}(x(t))
$$

The state matrices and input vectors of each T-S local linear model are:

$$
\begin{aligned}
& A_{1}=\left[\begin{array}{ccc}
2.31 & -71.83 & 227.6 \\
1 & 0 & 0 \\
0 & 1 & 0
\end{array}\right], B_{1}=\left[\begin{array}{l}
1 \\
0 \\
3
\end{array}\right] \\
& A_{2}=\left[\begin{array}{ccc}
1.604 & -107 & 180 \\
1 & 0 & 0 \\
0 & 1 & 0
\end{array}\right], B_{2}=\left[\begin{array}{c}
1 \\
0 \\
-3
\end{array}\right]
\end{aligned}
$$

One note that a common quadratic Lyapunov function to design the T-S fuzzy controller and then prove the stabilization [2] of the above closed loop T-S system does not exist.

From conditions (23) given in theorem 2 we obtain the following BMI (Bilinear Matrix Inequality) problem:

$$
\left\{\begin{array}{l}
\tilde{A}_{11}^{T} P_{1}+P_{1} \widetilde{A}_{11}<-Q \\
\tilde{A}_{22}^{T} P_{2}+P_{2} \widetilde{A}_{22}<-Q \\
\tilde{A}_{11}^{T} P_{2}+P_{2} \widetilde{A}_{11}+\widetilde{A}_{12}^{T} P_{1}+P_{1} \widetilde{A}_{12}+\widetilde{A}_{21}^{T} P_{1}+P_{1} \widetilde{A}_{21}<-Q \\
\tilde{A}_{22}^{T} P_{1}+P_{1} \widetilde{A}_{22}+\widetilde{A}_{21}^{T} P_{2}+P_{2} \widetilde{A}_{21}+\widetilde{A}_{12}^{T} P_{2}+P_{2} \widetilde{A}_{12}<-Q
\end{array}\right.
$$

with : $\widetilde{A}_{i j}=A_{i}-B_{i} K_{j}$

We know that BMI formulation is not convex problem and can have multiple local solutions. To linearize and solve these BMIs, we propose to decompose them on the following system :

$$
\left\{\begin{array}{l}
\widetilde{A}_{i i}^{T} P_{i}+P_{i} \widetilde{A}_{i i}<-Q \quad \forall i: 1,2 \\
\widetilde{A}_{i i}^{T} P_{j}+P_{j} \widetilde{A}_{i i}<0 \quad \forall i \neq j: 1,2 \\
\widetilde{A}_{12}^{T} P_{1}+P_{1} \widetilde{A}_{12}+\widetilde{A}_{21}^{T} P_{1}+P_{1} \widetilde{A}_{21}<-Q \\
\widetilde{A}_{21}^{T} P_{2}+P_{2} \widetilde{A}_{21}+\widetilde{A}_{12}^{T} P_{2}+P_{2} \widetilde{A}_{12}<-Q
\end{array}\right.
$$

Global optimality is ensured through the convex reformulation of the problem. This convexity is achieved through the matrix change of variables:

$$
P_{i}^{-1}=X_{i}, Y_{i j}=K_{i} X_{j}, S_{i}=X_{i} Q X_{i}
$$

where: $X_{i}=X_{i}^{T}>0 \quad \forall i, j: 1,2$

Constraints (26) can be shown equivalent to the six following LMIs constraints:

$$
\begin{array}{lr}
X_{i} A_{i}^{T}+A_{i} X_{i}-Y_{i i}^{T} B_{i}^{T}-B_{i} Y_{i i}<-S_{i} & i: 1 . .2 \\
X_{i} A_{j}^{T}+A_{j} X_{i}-Y_{j i}^{T} B_{j}^{T}-B_{j} Y_{j i}<0 & i \neq j: 1 . .2 \\
X_{i}\left(A_{i}^{T}+A_{j}^{T}\right)+\left(A_{j}+A_{i}\right) X_{i}-Y_{i i}^{T} B_{j}^{T}-B_{j} Y_{i i} \\
-Y_{j i}^{T} B_{i}^{T}-B_{i} Y_{j i}<-S_{i} \quad i \neq j: 1 . .2
\end{array}
$$

The feedback gain $K_{i}$, matrix $P_{i}$ and a common matrix $Q$ can be obtained as:

$$
P_{i}=X_{i}^{-1}, K_{i}=Y_{i j} P_{j}, Q=P_{i} S_{i} P_{i}
$$

Solving the LMIs system (27), we obtain :

$$
\begin{aligned}
P_{1} & =\left[\begin{array}{ccc}
2.1 & 7.6 & 33.8 \\
7.6 & 142.1 & 146.8 \\
33.8 & 146.8 & 1136.8
\end{array}\right], P_{2}=\left[\begin{array}{ccc}
3.3 & 9.8 & 68.8 \\
9.8 & 155.5 & 201.7 \\
68.8 & 201.7 & 2320.6
\end{array}\right] \\
Q & =10^{3}\left[\begin{array}{ccc}
0.84 & 3.04 & 13.52 \\
3.04 & 56.84 & 58.72 \\
13.52 & 58.72 & 454.72
\end{array}\right]
\end{aligned}
$$




$$
\begin{aligned}
K_{1} & =\left[\begin{array}{lll}
7.39 & 36.07 & 205.38
\end{array}\right], \\
K_{2} & =\left[\begin{array}{lll}
-8.34 & -24.36 & -279.52
\end{array}\right]
\end{aligned}
$$

The inequality (24) allows to compute the upper bound of the rate of variation of state variables guaranteeing the exponential stabilization of the global closed loop T-S fuzzy systems.

$$
\|\dot{x}(t)\| \leq 109.40
$$

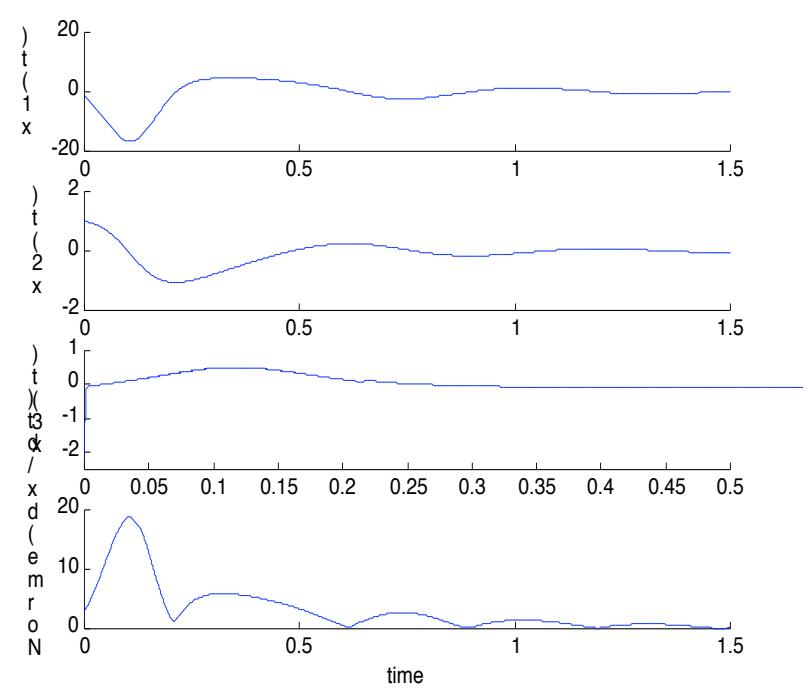

Figure 2: States trajectories with initials values $x(0)=\left[\begin{array}{lll}-1 & 1 & -2\end{array}\right]$

\section{Conclusion}

The main originality of the proposed method concerns, on one hand, the reduction of the number of constraints guaranteeing the stability of the global system and, on the other hand, the laying down of a systematic method for computing upper bounds of the rates of variation of state variables guaranteeing the stability and the stabilization of T-S continuous fuzzy systems.

We have presented new relaxed stability conditions and LMI-based designs for continuous fuzzy control systems. More precisely, the stability is proven if the rates of variations of the states are bounded. This class of Lyapunov function is substantially richer than the globally quadratic functions. By exploiting the non-quadratic stability of T-S continuous fuzzy systems and solving a convex optimisation problem formulated as a set of LMIs, we have shown how to pick systematically upper bounds of the variation of the state to guarantee the stability of the global T-S continuous fuzzy model. These conditions may be used to design fuzzy regulators and/or fuzzy observers.

\section{References}

[1] A. Jadbabaie "A reduction in conservatism in stability and L2 Gain analysis of T-S fuzzy systems via Linear matrix inequalities "IFAC 1999, $14^{\text {th }}$ triennial World congress, Beijing, P.R. China. pp. 285-289.

[2] T. Takagi, T. Ikeda, H. O. Wang, "Fuzzy regulators and fuzzy observers: relaxed stability conditions and LMI-based design" IEEE Trans. on Fuzzy Systems, vol. 6, no. 2, pp. 250-256, 1998.

[3] M. Johansson, A. Rantzer, K. Arzén "Piecewise quadratic stability for affine Sugeno systems", FUZZ. IEEE'98, Anchorage, Alaska, 1998.

[4] K. Tanaka, T. Ikeda, H. O. Wang, "Robust stabilisation of uncertain non-linear systems via fuzzy control: quadratic stability, $\mathrm{H}$ control theory, and LMIs" IEEE Trans. on Fuzzy Systems, vol. 4, no. 1. pp 1-12, 1996.

[5] T. Takagi, M. Sugeno, " Fuzzy identification of systems and its application to modelling and control" IEEE Trans. on Systems, Man, Cybernetics, vol. 15, no.1, pp. 116-132,1985.

[6] K. Tanaka, M. Sugeno, "Stability and design of fuzzy control systems " Fuzzy Set and Systems, vol. 45, no. 2, pp 135-156. 1992.

[7] H. O. Wang, K. Tanaka, M. F. Griffin “An approach to fuzzy control of nonlinear systems: stability and design: issues" IEEE. Trans. on Fuzzy Systems, vol. 4, no.1, 1996.

[8] J. Castro " Fuzzy logic controllers are universal approximator" IEEE Trans. on Systems, Man, Cybernetics, vol. 25, pp. 629-635, April 1995.

[9] K. Tanaka, M. Nishimuna, H.O. Wang, “ Multiobjective fuzzy control of high rise/high speed elevators using LMIs" American Control Conference, Philadelphia, Pennsylvanie, pp. 3450-3454, June 1998.

[10] S. Boyd et al. "Linear matrix inequalities in systems and control theory" Philadelphia, PA: SIAM, 1994.

[11] M. C. M. Teixeira, S. H. Zak, " Stabilizing controller design for uncertain nonlinear systems using fuzzy models" IEEE. Trans. on Fuzzy Systems, vol.7, no.2, pp. 133-140, 1999.

[12] K. S. Narendra, J. Balakrishnan, “ A common Lyapunov function for stable LTI systems with commuting A-matrices "IEEE Trans. on Automatic Control, vol. 39, no. 12, pp. 2469-2471, 1994.

[13] J.-S. Chai, S. Tan, Q. Chan, C.-C. Hang, " A general fuzzy control scheme for nonlinear processes with stability analysis" Fuzzy Sets and Systems 100, pp. 179-195, 1998.

[14] A. Weinmann, Uncertain models and robust control, Springer-Verlag, Wien, 1991. 